\title{
Up-regulation of Hsp72 and keratin16 mediates wound healing in streptozotocin diabetic rats
}

\author{
Rasha R. Ahmed ${ }^{1}$, Ayman Mahmoud², Osama M. Ahmed², Ali Metwalli,4,4 and Hossam Ebaid ,6* $^{5 *}$
}

\begin{abstract}
Background: Impaired wound healing is a complication of diabetes and a serious problem in clinical practice. We previously found that whey protein (WP) was able to regulate wound healing normally in streptozotocin (STZ)-diabetic models. This subsequent study was designed to assess the effect of WP on heat shock protein-72 (Hsp72) and keratin16 (Krt16) expression during wound healing in diabetic rats.
\end{abstract}

Methods: WP at a dosage of $100 \mathrm{mg} / \mathrm{kg}$ of body weight was orally administered daily to wounded normal and STZdiabetic rats for 8 days.

Results: At day 4, the WP-treated diabetic wound was significantly reduced compared to that in the corresponding control. Diabetic wounded rats developed severe inflammatory infiltration and moderate capillary dilatation and regeneration. Treated rats had mild necrotic formation, moderate infiltration, moderate to severe capillary dilatation and regeneration, in addition to moderate epidermal formation. Hsp72 and Krt16 densities showed low and dense activity in diabetic wounded and diabetic wounded treated groups, respectively. At day 8, WP-treatment of diabetic wounded animals revealed great amelioration with complete recovery and closure of the wound. Reactivity of Hsp72 and Krt16 was reversed, showing dense and low, or medium and low, activity in the diabetic wounded and diabetic wounded treated groups, respectively. Hsp72 expression in the pancreas was found to show dense reactivity with WPtreated diabetic wound rats.

Conclusion: This data provides evidence for the potential impact of WP in the up-regulation of Hsp72 and Krt16 in T1D, resulting in an improved wound healing process in diabetic models.

Keywords: Diabetic model, Whey protein, Heat shock protein-72, Keratin16, Wound healing

\section{Background}

Impaired diabetic wound healing occurs as a consequence of excessive reactive oxygen species (ROS) and inflammatory cytokine production [1-3]. Intracellular heat shock proteins (Hsps) are upregulated in cells subjected to stressful stimuli, including inflammation and oxidative stress, and exert a protective effect against hypoxia, excess oxygen radicals, endotoxin, infections and fever [4]. Recent studies have confirmed that Hsps support antioxidant [5], anti-inflammatory [6] or

\footnotetext{
*Correspondence: hossamebaid@yahoo.com

${ }^{5}$ Department of Zoology, College of Science, King Saud University,

P.O. Box 2455, Riyadh 11451, KSA

Full list of author information is available at the end of the article
}

immune enhancing [7] therapies in sepsis. Park et al. [8] found that Hsp72 functions as an endogenous inhibitor of apoptosis signal-regulating kinase 1 . It plays a role in many cellular activities, including prevention of cell death initiated by various apoptotic stresses, such as ionizing irradiation and TNF-a $[9,10]$, by suppressing apoptotic signalling pathways, including caspase cascades [11] and the JNK signalling pathway [12].

Keratins are the most prominent cytoskeletal proteins in keratinocytes [13]. Keratin 16 (Krt16) is usually referred to as an activation- and hyperproliferation-associated keratin [14]. Although, Krt16 impacts cell migration by interacting with Src kinase [15], its induction significance in response to environmental stressors in 
epithelial cancers and in chronic inflammatory disorders [16] is largely unknown. Juliane et al. [17] showed that when the epidermal barrier is experimentally challenged by acute pro-inflammatory and mechanical stimuli, keratinocytes lacking Krt16 fail to regulate the production of innate danger signals properly, and over-activate the expression of cytokines and other regulators of skin barrier function.

Whey protein (WP) has been shown to be able to regulate impaired wound healing normally [18]. Here we aimed to find a correlation between the achievement of normal healing in diabetic wounds by WP and the regulation of two important markers, Hsp72 and Krt16.

\section{Methods}

After shaving and disinfecting the rat dorsal skin surface, a skin wound site was made using a Biopsy Punch $(5 \mathrm{~mm}$ in the diameter).

Rats, at the end of each tested period, were euthanatized and $10 \times 10 \mathrm{~mm}$ of skin including the wound site and pancreas were excised then fixed in $10 \%$ neutral formalin solution. After washing with distilled water, the paraffin embedding specimens were developed in the routine method. Immunohistochemical, and hematoxylin \& eosin (HE) staining were carried out on $4 \mu \mathrm{m}$-thick sections.

\section{Measurement of wound diameter and closure}

The wound diameter was measured on days 1,4 and 8 after incision and percentage of wound closure was calculated using the following formula: wound closure rate on day $\mathrm{X}(\%)=[($ wound diameter on day $0-$ wound diameter on day $\mathrm{X}) /($ wound diameter on day 0$)] \times 100$.

\section{Ethical approval and preparation of un-denatured camel milk whey proteins}

Camel milk was obtained from a camel breed (Majaheem) from the Najd region (Alazeria farm; GPS: 30002 47/300 02 27) in Saudi Arabia. Specific permissions were not required for activities in this private farm. This study did not involve endangered or protected species. Regarding experimental animals, all procedures were conducted in accordance with the standards set forth in the guidelines for the care and use of experimental animals by the Committee for the Purpose of Control and Supervision of Experiments on Animals and the National Institutes of Health. The study protocol (care and handling of experimental animals) was approved by the Animal Ethics Committee of the Zoology Department in the College of Science at King Saud University.

The milk was skimmed by centrifugation at $5000 \mathrm{~g}$ for 20 min using an IEC Model K centrifuge (Boston, USA). Skim milk was acidified to $\mathrm{pH} 4.3$ using $1 \mathrm{M}$ of $\mathrm{HCl}$. The precipitated casein was removed by centrifugation, and the supernatant containing the whey protein was saturated with ammonium sulfate (70\% saturation) and incubated overnight at $4{ }^{\circ} \mathrm{C}$. The precipitated whey proteins were collected by centrifugation and dialyzed against distilled water for $48 \mathrm{~h}$ at $4{ }^{\circ} \mathrm{C}$ using a Spectra/Pro ${ }^{\circledR} \mathrm{Mem}$ brane, MWCO 6000-8000 Da. The obtained dialyzate was lyophilized using a Unitop 600 SL, (Virtis Company, Gardiner, New York 12525 USA) and were kept at $-20{ }^{\circ} \mathrm{C}$ until use. The dialyzate containing un-denatured whey proteins were freeze-dried and refrigerated until use.

\section{Diabetic models}

Diabetes was induced by a single injection of freshly dissolved STZ (60 mg/kg of body weight; Sigma, USA) in a $0.1 \mathrm{~mol} / \mathrm{l}$ citrate buffer $(\mathrm{pH} 4.5)$ into the peritoneum. Control rats were injected with citrate buffer. Seven days after STZ injection, the rats were screened for serum glucose levels. Rats with a serum glucose level $\geq 200 \mathrm{mg} / \mathrm{dl}$ after $2 \mathrm{~h}$ of glucose intake were considered diabetic and selected for further studies.

\section{Experimental design}

The supplemented volume for all groups was constant and did not exceed $250 \mu \mathrm{l}$ per dosage per day. The optimal dose of WP was determined in our laboratory on the basis of the LD50 and several established studies and parameters. The animals were allocated into 6 groups of 12 animals each, assigned as follows:

1. Uninjured control group that were orally supplemented with distilled water $(250 \mu \mathrm{l} / \mathrm{rat} /$ day $)$.

2. Wounded non-diabetic group with daily administration of the vehicle ( $250 \mu \mathrm{l} / \mathrm{rat} /$ day), $1 \%$ carboxymethyl cellulose $(\mathrm{CMC})$, by gastric intubation for 4 days or by gastric intubation for 8 days.

3. Wounded non-diabetic group with daily administration of WP at $100 \mathrm{mg} / \mathrm{kg}$ of body weight $(250 \mu \mathrm{l} /$ rat/day), dissolved in $1 \% \mathrm{CMC}$, by gastric intubation either for 4 days or for 8 days.

4. Uninjured diabetic group (non-wounded diabetic: non-wounded D) that were orally supplemented with distilled water ( $250 \mu \mathrm{l} / \mathrm{rat} /$ day).

5. Wounded diabetic group with daily administration of $1 \% \mathrm{CMC}(250 \mu \mathrm{l} / \mathrm{rat} /$ day $)$ by gastric intubation for 4 days or by gastric intubation for 8 days.

6. Wounded diabetic group with daily treatment of WP at $100 \mathrm{mg} / \mathrm{kg}$ of body weight $(250 \mu \mathrm{l} / \mathrm{rat} /$ day $)$ by gastric intubation either for 4 days or for 8 days.

\section{Histological analyses}

After fixation with $4 \%$ paraformaldehyde for $24 \mathrm{~h}$ at room temperature, the specimens were embedded in 
paraffin and sectioned in a plane perpendicular to the incision. Sections $5 \mu \mathrm{m}$ thick were mounted on slides, dewaxed, rehydrated to distilled water, and stained with HE. For each group, three sections of three different animals were randomly selected for histological evaluation. The mean value was used for statistical comparison.

\section{Immunohistochemical study}

The streptavidin-biotin-peroxidase technique was used for tests with anti-Hsp72 (Catalog No. SPA810, Stressgen, USA), five-micrometer-thick sections were de-waxed and rehydrated in a descending series of alcohols. Antigen retrieval by microwave and citrate buffer ( $\mathrm{pH} 6$ ) was performed, using the procedure specified by the antibody manufacturer. Slides were subjected to endogenous tissue peroxidase blocking. Incubation was performed with the primary antibody at a dilution of $1: 1000$ in PBS/0.1 \% Tween. The samples were then incubated with a biotinylated swine-antirabbit/goat antibody, as well as a streptavidin-biotin-peroxidase conjugate (LSAB System, Dako ${ }^{\circledR}$, Carpenteria, CA, USA) for 30 min each. The reaction was then revealed by diaminobenzidine $\left(\right.$ Dako $\left.^{\circledR}\right)$, and the sections were dehydrated in an increasing series of alcohols, immersed in xylol, and mounted in resin for conventional light microscopy. For the negative control, sections were incubated in a buffer without primary antibody.

Additional immuno-histochemical reactions against cytokeratin 16 were performed. Primary antibodies of

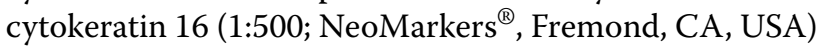
were used after antigen retrieval (citric acid in water bath at $100{ }^{\circ} \mathrm{C}$ for $45 \mathrm{~min}$ for cytokeratin 16). Incubation and reaction of secondary antibodies were the same as those described for Hsp.

\section{Evaluation of the intensity of immunohistochemical staining}

All expression patterns were analyzed by two independent investigators experienced in skin and pancreas histology. The expression densities of Hsp72 in skin and pancreas and krt16 ranged from $0=$ no positive cells (undetected), $1=$ low density $(<10 \%$ positive cells $)$, $2=$ medium density $(10-25 \%$ positive cells), $3=$ dense (26-70 \% positive cells), $4=$ very dense $(71 \%$ positive cells) as described previously by Souil et al. [19]

\section{Statistical analysis}

The data of wound diameter were analyzed using oneway analysis of variance (ANOVA) followed by LSD test to compare various groups with each others according our previously publication [2].

\section{Results}

\section{Wound closure}

The diameter of the wound site in each of the groups was measured 1, 4 and 8 days after the incision (Fig. 1; Table 1). In control animals, the diameter of the wound reduced to $4.6 \pm 0.13 \mathrm{~mm} 1$ day post-incision and to $3.8 \pm 0.1 \mathrm{~mm} 4$ days post-incision and $3 \pm 0.1 \mathrm{~mm}$ recording wound closure percent of 8.0, 24.0 and $40 \%$ respectively. This closure percent was greatly accelerated after treatment as compared to the control wounded animals where the diameter of the wound reduced to $4.2 \pm 0.15 \mathrm{~mm} 1$ day post-incision and to $2.5 \pm 0.0 \mathrm{~mm}$ 4 days post-incision recording wound closure percent of 16.0 and 50.0 respectively. Eight days post-incision, the wound site was covered by epidermis recording wound closure percent of $100 \%$. In diabetic animals, the wound site reduced to $4.8 \pm 0.1 \mathrm{~mm} 1$ day after experiment, to $4.4 \pm 0.13 \mathrm{~mm}$ at 4 days after, and to $3.6 \pm 0.18 \mathrm{~mm}$ 8 days after; the recorded wound closure percent were calculated to be 4.0, 12.0 and 28.0 respectively. In diabetic treated rats, the wound was reduced to $4.4 \pm 0.12 \mathrm{~mm}$

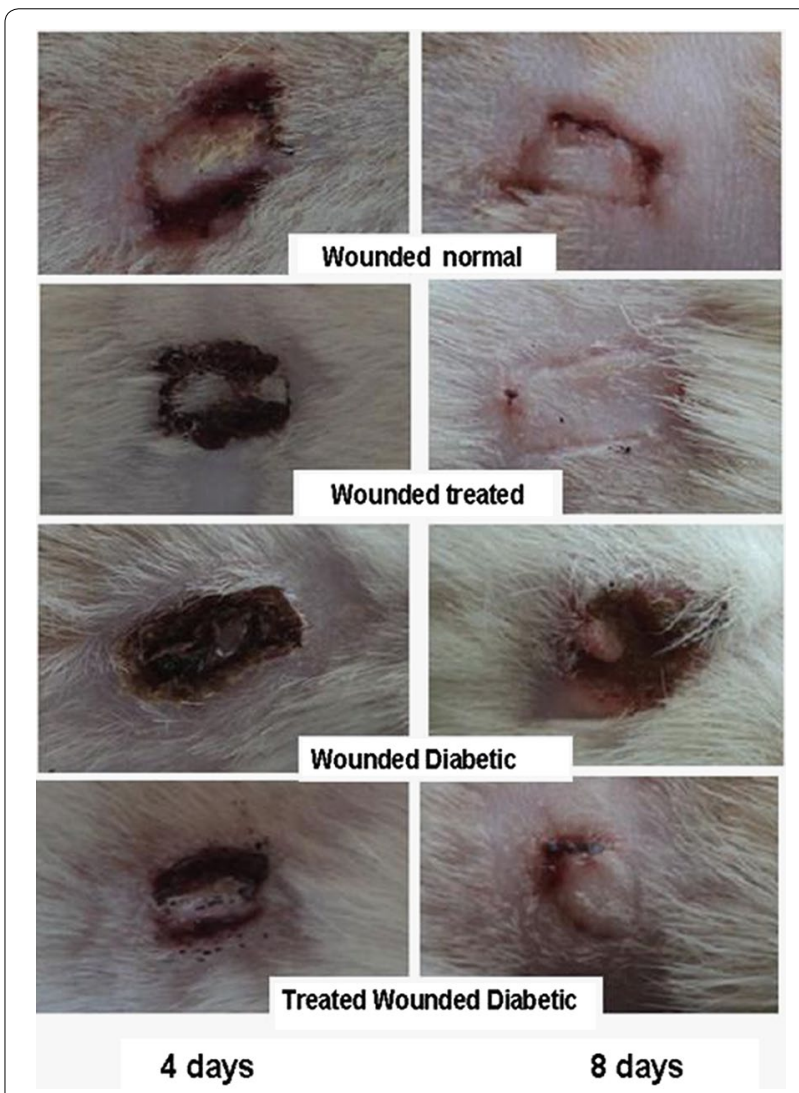

Fig. 1 Progress of cutaneous wound healing in control wounded, control wounded treated, diabetic wounded control and diabetic wounded treated groups 4 days (right column) and 8 days (left column) after incision (from Ebaid et al. [2]). $\times 4$ 
Table 1 Effect of treatment with whey protein on wound diameter and closure percent of normal wounded and diabetic wounded rats

\begin{tabular}{|c|c|c|c|c|c|c|}
\hline \multirow[t]{2}{*}{ Groups } & \multicolumn{2}{|l|}{1 day } & \multicolumn{2}{|l|}{4 days } & \multicolumn{2}{|l|}{8 days } \\
\hline & Mean & $\%$ & Mean & $\%$ & Mean & $\%$ \\
\hline Normal wounded & $4.6 \pm 0.13^{\mathrm{ab}}$ & 8 & $3.8 \pm 0.1^{\mathrm{ef}}$ & 24 & $3.0 \pm 0.1^{\mathrm{g}}$ & 40 \\
\hline Normal wounded treated & $4.2 \pm 0.15^{c d}$ & 16 & $2.5 \pm 0.0^{h}$ & 50 & $0.0 \pm 0.0^{i}$ & 100 \\
\hline Diabetic wounded & $4.8 \pm 0.1^{a}$ & 4 & $4.4 \pm 0.13^{b c}$ & 12 & $3.6 \pm 0.18^{f}$ & 28 \\
\hline Diabetic wounded treated & $4.4 \pm 0.12^{b c}$ & 12 & $4.0 \pm 0.0^{\text {de }}$ & 20 & $3.2 \pm 0.13^{g}$ & 36 \\
\hline
\end{tabular}

Data are expressed as mean \pm SE. Number of samples in each group is 3

Means which share the same superscript symbol(s) are not significantly different

F-probability: $p<0.001$; LSD at the $5 \%$ level $=0.201$; LSD at the $1 \%$ level $=0.273$

1 day post-incision, to $4.0 \pm 0.0 \mathrm{~mm} 4$ days after, and to $3.2 \pm 0.13 \mathrm{~mm} 8$ days after the operation recording wound closure percent $12.0,20.0$ and 36.0 respectively. The diameter of both normal wounded treated group and diabetic wounded group was significantly decreased at 1 , 4 and 8 days after wounding as compared to their corresponding controls.

\section{Histological analyses}

The histopathological findings of skin are shown in Figs. 2 and 3.

\section{One day after incision}

In both wounded non-diabetic animals and diabetic animals that had been wounded but not treated, the wound consisted of a large (scab) uncovered by epidermis. In the wounded treated animals, the wound was filled with necrotic debris and fibrin (crusts) and has proliferating granulation tissue. The dermis showed oedema and vascular congestion. These changes were less apparent in the diabetic un-treated animals than in the non-diabetic control group.

\section{Four days after incision}

The wound site was diminished due to deep contraction but was still lined by granulation tissue and covered with crusts. The dermis cellularity increased, mainly due to the proliferation of fibroblasts and new matrix deposition. The epidermis was thicker on the margin of the wound (hyperplasia) and was starting to cover the defect. Non-diabetic wounded rats and diabetic animals that had been wounded but not treated developed severe inflammatory infiltration and moderate capillary dilatation and regeneration. All these changes were slightly more evident in diabetic untreated rats compared to the diabetic untreated groups. In contrast, treated rats had mild necrotic formation, moderate infiltration, moderate to severe capillary dilatation and regeneration, in addition to moderate epidermal formation.

\section{Eight days after incision:}

At day 8, in the wounded animals (both diabetic and non-diabetic) the wound was completely lined by hyperplastic epidermis, but the scar surface was reduced due to wound contraction and matrix deposition. Some immature collagen fibres still remained at the centre. Few inflammatory mononuclear cells remained at the border of the scar. Treatment of both groups revealed a great amelioration of these changes with complete recovery and closure of the wound in the non-diabetic treated group.

\section{The histopathological changes in the pancreas}

In the diabetic group, a decrease in pancreatic islet numbers and size, atrophy and vacuolation, and invasion of connective tissues in the parenchyma of pancreatic islets were detected after 8 days, but, compared to the diabetic group, these abnormal histological signs dramatically decreased in the group treated with the extract. In contrast, these changes became much more severe in the diabetic wounded groups four and eight days after incision. Treatment of these two latter groups greatly ameliorated these changes.

\section{Immunohistochemical findings}

\section{Skin}

Keratin 16 The intensity of Krt16 in the tested groups and periods are shown in Fig. 4 and Table 2.

Krt16 is not expressed in healthy epidermal skin. Its reaction is restricted to epidermal cells and dermal glands. The reactivity was medium in both normal wounded and normal wounded treated groups while it showed low and dense activity in diabetic wounded and diabetic wounded treated animals 4 days after wound incision. 

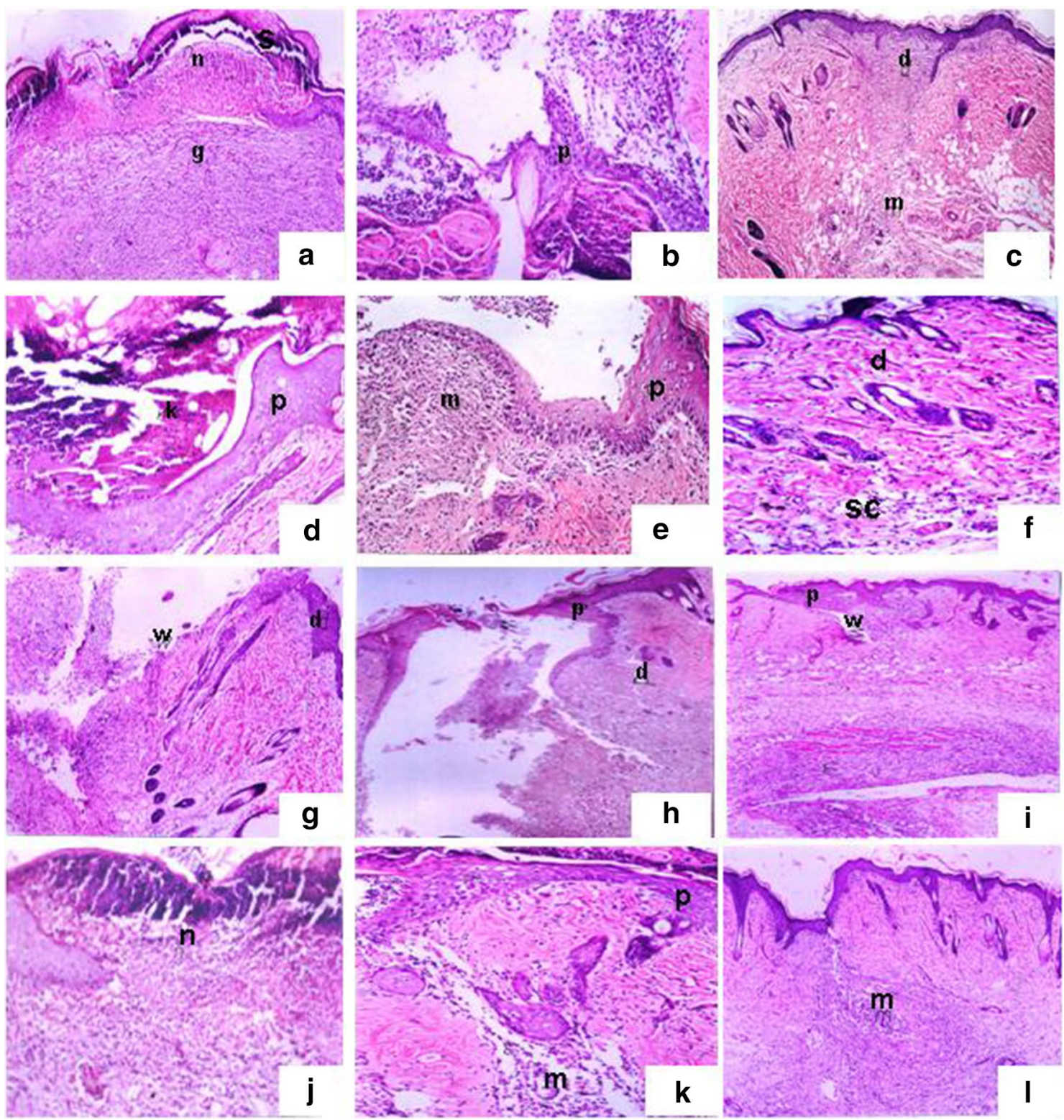

Fig. 2 Histological changes during the wound-healing process on the first (left), fourth (centre) and eighth (right) days after incision in control wounded $(\mathbf{a}-\mathbf{c})$, control wounded treated $(\mathbf{d}-\mathbf{f})$, diabetic wounded control $(\mathbf{g}-\mathbf{i})$ and diabetic wounded treated $(\mathbf{j}-\mathbf{I})$ groups; scab $(s)$, granulation $(g)$, dermis $(d)$, wound $(\boldsymbol{W})$, keratinization $(K)$, epidermis $(p)$, necrosis $(n)$ and inflammatory cells $(m) . \times 100$

Eight days after incision, low, no, medium and low reactivity were recorded respectively in normal wounded, normal wounded treated, diabetic wounded and diabetic wounded treated groups.

Hsp72 The densities of Hsp72 and reactivity in all tested groups and periods are shown in Fig. 5 and Table 3.

No difference was recorded in the expression densities of cells positive for Hsp72 in any of the tested groups one day after incision. Four days after wound incision, however, Hsp72 densities were dense, very dense, medium and dense in normal wounded, normal wounded treated, diabetic wounded and diabetic wounded treated groups respectively. This activity was expressed throughout all the layers of the epidermis and dermal glands. Melanocytes, fibroblasts and other epidermal cells were negative, though (Fig. 6).

Eight days after incision, the reactivity in the same kinds of cells were recorded and showed low, no, dense and low activity in normal wounded, normal wounded 


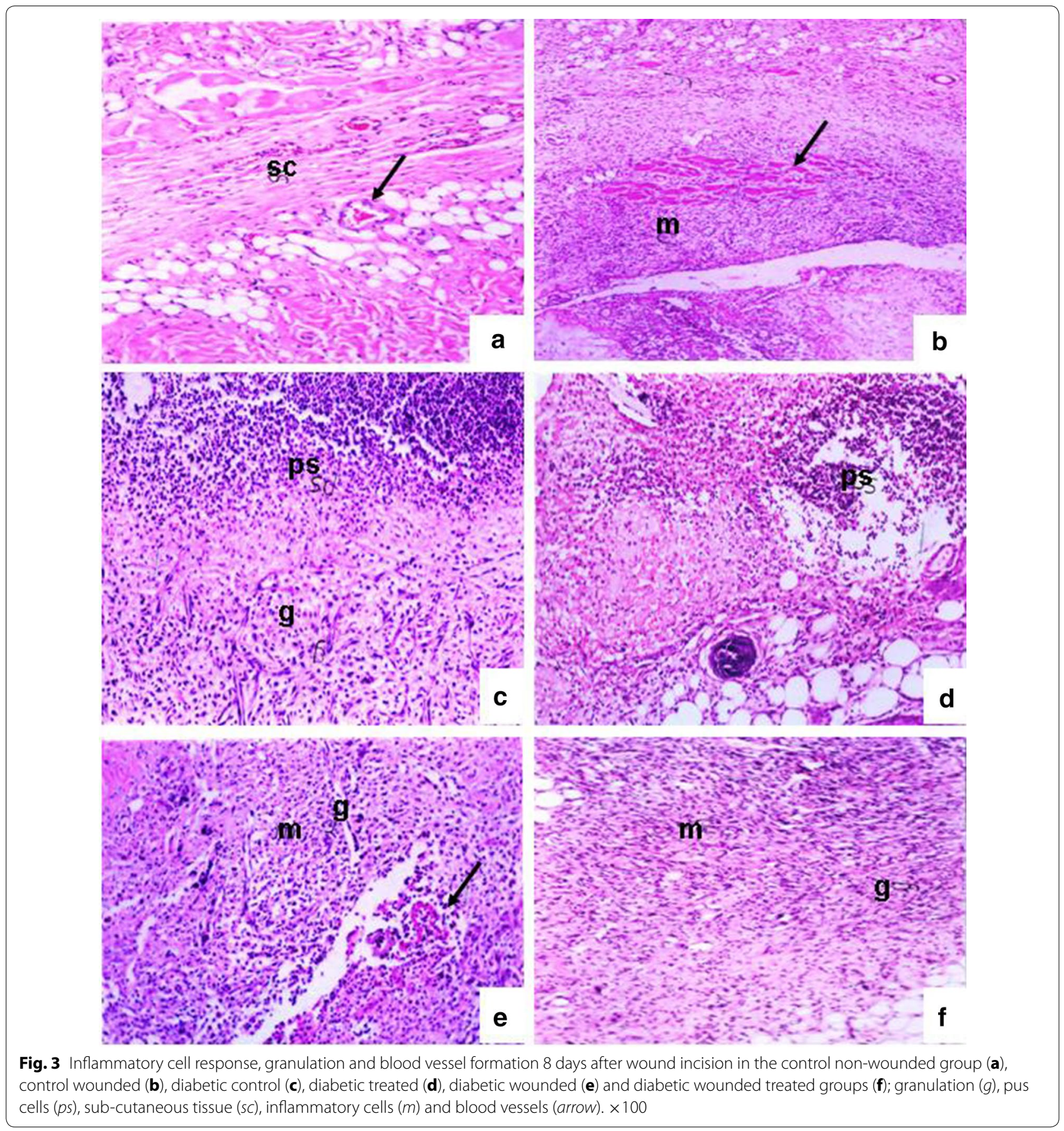

treated, diabetic wounded and diabetic wounded treated groups, respectively.

\section{Pancreas}

The densities of Hsp72 and reactivity in all tested groups and periods are shown in Fig. 7 and Table 4.
Hsp72 expression densities showed no difference in any of the tested groups one day after incision. Four days after wound incision, however, Hsp72 showed dense and mild reactivity in normal wounded and normal wounded treated groups. Hsp72 reactivity was absent or low in the remaining groups, however. The reactivity was mainly 


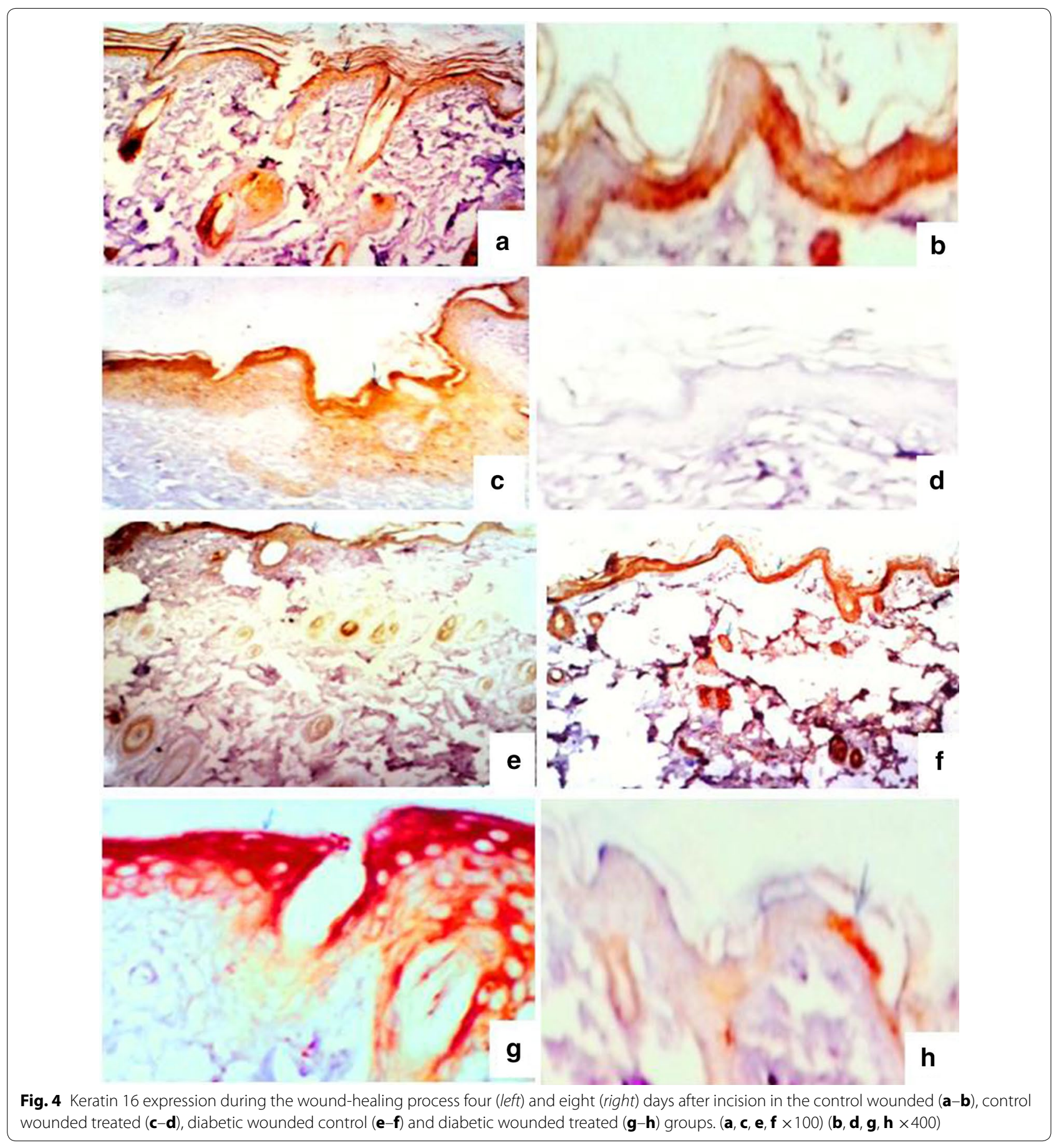

recorded in pancreatic islets but it was also noticed in the exocrine part of the control wounded group.

Eight days after incision, the reactivity was noticed in the same types of cells, revealing low reactivity in the normal wounded group and a low response in the diabetic and diabetic wounded animals, as well as low, mild and dense reactivity in the wounded treated, diabetic-treated and diabetic wounded treated groups respectively. 
Table 2 The densities of Krt16 and reactivity in all tested groups and periods

\begin{tabular}{lll}
\hline Keratin Krt16 & 4 days & $\mathbf{8}$ days \\
\hline Normal wounded & Medium & Low \\
Normal wounded treated & Medium & None \\
Diabetic wounded & Low & Medium \\
Diabetic wounded treated & Dense & Low \\
\hline
\end{tabular}

\section{Discussion}

Diabetes decreases immune response capacity, including the suppression of immune cell function and is a major public health concern [20]. Many studies have been performed to assess the potential utility of natural products as immunomodulatory agents to enhance host responses to disease [21]. Although, we have previously proved that WP was able to return wound healing impaired by
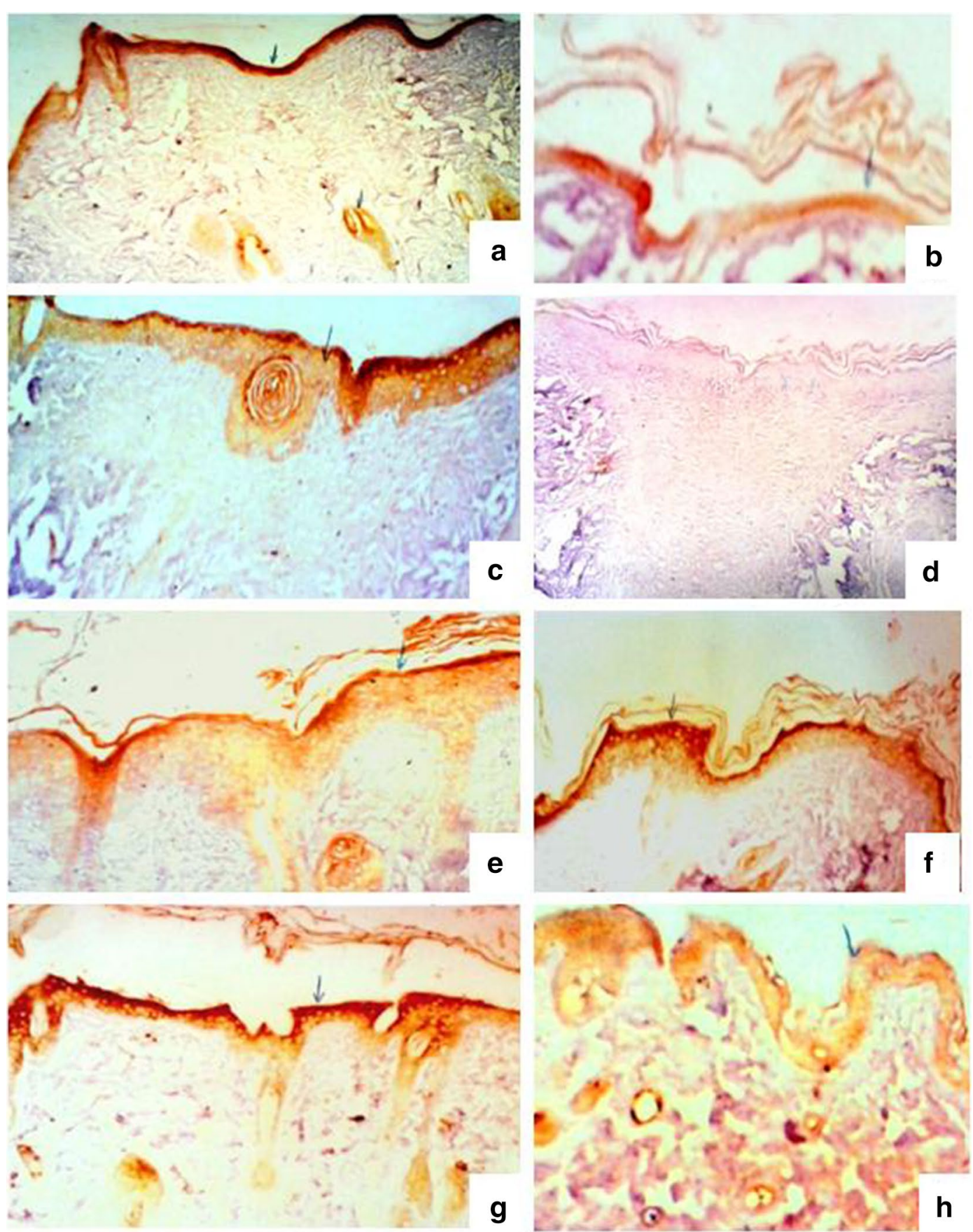

Fig. 5 Heat shock protein 72 expression during the wound-healing process four (left) and eight (right) days after incision in the control wounded $(\mathbf{a}-\mathbf{b})$, control wounded treated $(\mathbf{c}-\mathbf{d})$, diabetic wounded control $(\mathbf{e}-\mathbf{f})$ and diabetic wounded treated $(\mathbf{g}-\mathbf{h}) \mathrm{groups} \times 100$ 
Table 3 The densities of Hsp72 and reactivity in all tested groups and periods

\begin{tabular}{lll}
\hline HSP72 & 4 days & $\mathbf{8}$ days \\
\hline Normal wounded & Dense & Low \\
Normal wounded treated & Very dense & No \\
Diabetic wounded & Medium & Dense \\
Diabetic wounded treated & Dense & Low \\
\hline
\end{tabular}

diabetes to a more normal pattern [1-3], the exact mechanism by which this occurs is still poorly investigated. In this study we have explored the role of Hsp72 and Krt16 in accelerating and normalizing impaired diabetic wound healing in rodent-models.

Hsps play a critical role in the overall process of wound repair [22]. We found that Hsp72, in normal wounds, was highest at day 4, before gradually decreasing during subsequent healing phases. WP was found to induce a similar behaviour of Hsp72 in wounds under the stress of diabetes. Similarly, Hsp72 expression was found to be highest starting from day 3 post-intervention in the spinocellular layer leading to an up-regulation of Hsp47 [23]. Hsp72's role is as an indicator of cellular stress and injury [22]. Generally, Hsps are chaperone proteins preventing cells from undergoing apoptosis and ensuring their cellular function [24].
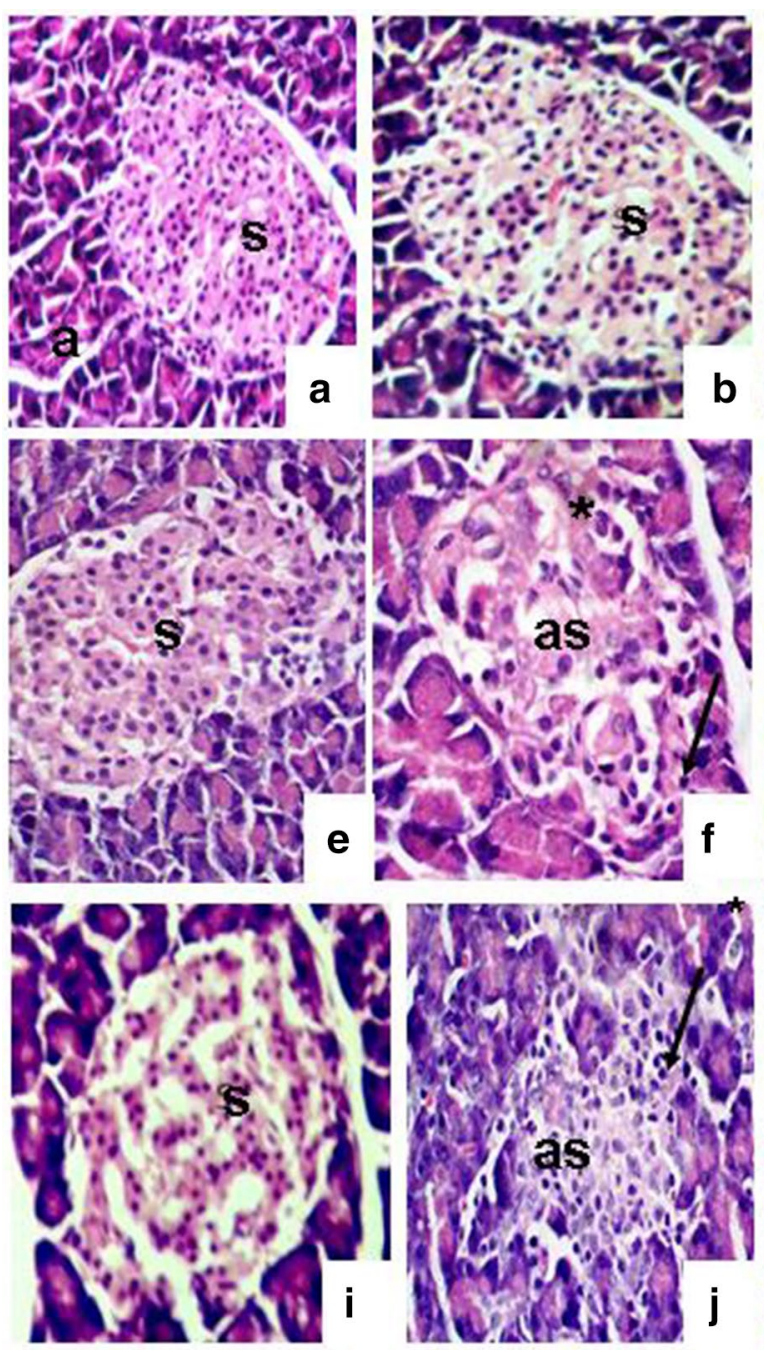
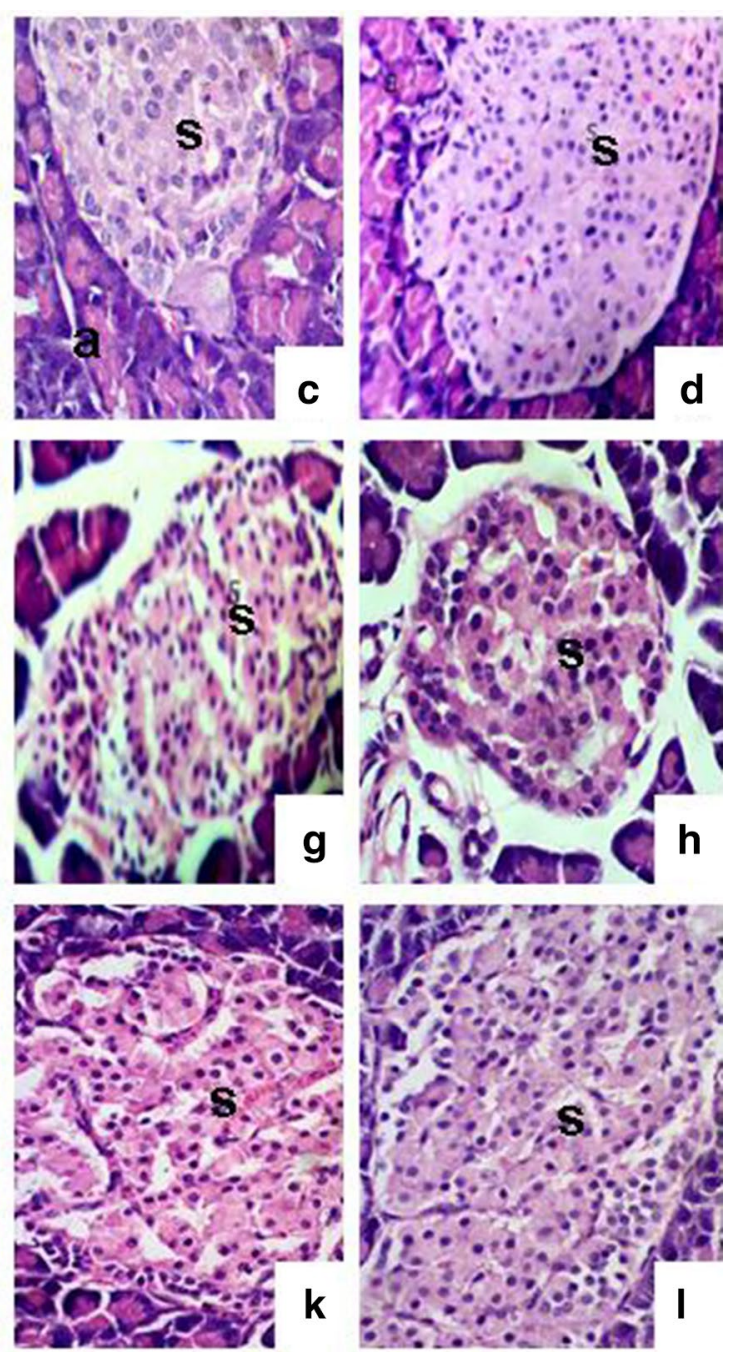

Fig. 6 Histological changes during the wound-healing process on the fourth and eighth days, respectively, after incision of the control wounded non-treated $(\mathbf{a}-\mathbf{b})$, control wounded treated $(\mathbf{c}-\mathbf{d})$, diabetic control non-treated $(\mathbf{e}-\mathbf{f})$, diabetic control treated ( $\mathbf{g}-\mathbf{h})$, diabetic wounded control $(\mathbf{i}-\mathbf{j})$ and diabetic wounded treated (k-I) groups; Islands of Langerhans (s), atrophied islands (as) lymphocytic infiltration (arrow) and connective tissue invasion in the parenchyma (asterisk). $\times 400$ 

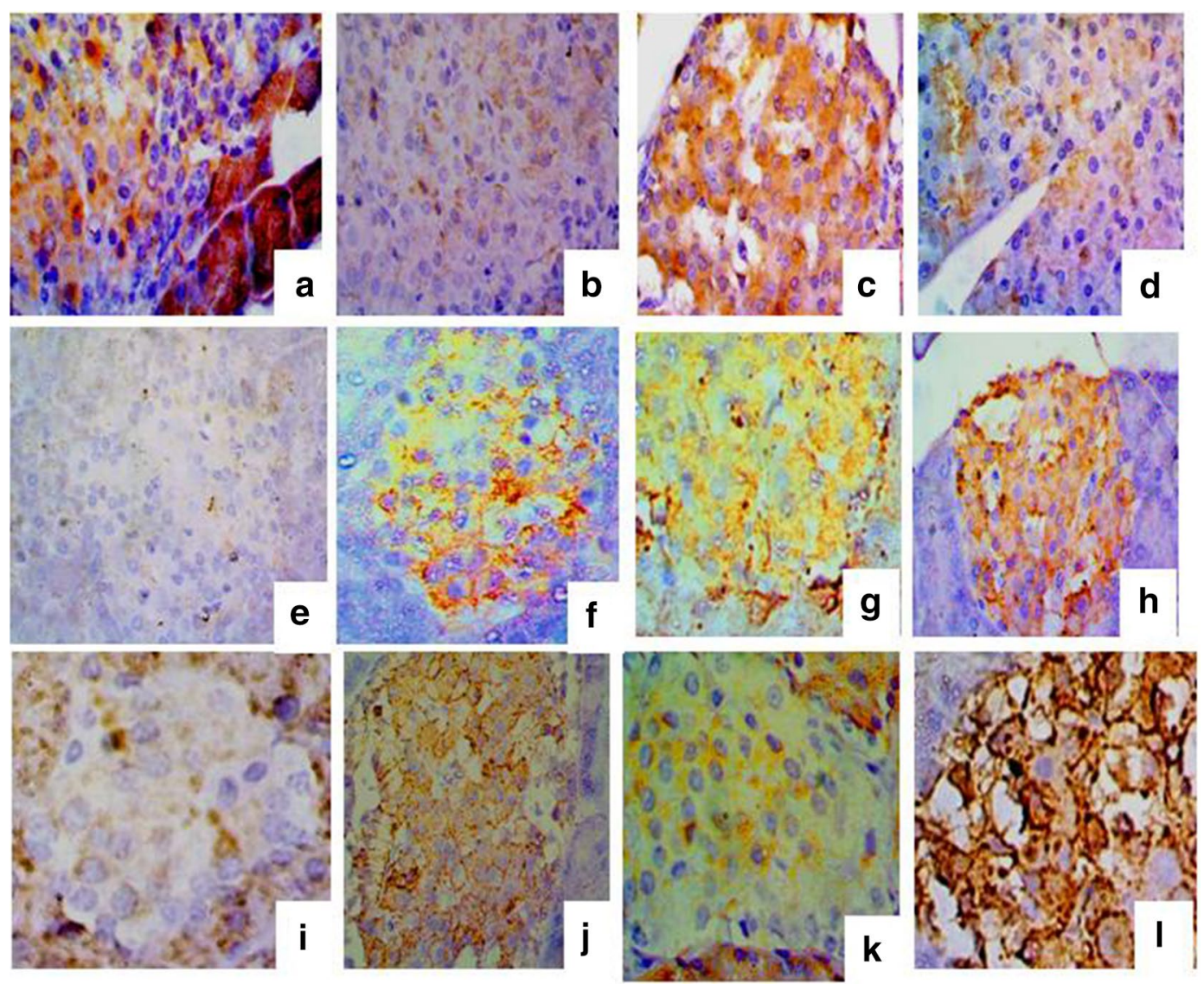

Fig. 7 Heat shock protein 72 expression during the wound-healing process four and eight days after incision in the control wounded (a-b), control wounded treated $(\mathbf{c}-\mathbf{d})$, diabetic non-wounded control $(\mathbf{e}-\mathbf{f})$, diabetic non-wounded treated $(\mathbf{g}-\mathbf{h})$, diabetic wounded control $(\mathbf{i}-\mathbf{j})$ and diabetic wounded treated $(\mathbf{k}-\mathbf{I})$ groups. $\times 400$

Table 4 The densities of Hsp72 and reactivity in all tested groups and periods

\begin{tabular}{lll}
\hline HSP72 & 4 days & $\mathbf{8}$ days \\
\hline Normal wounded & Dense & No \\
Normal wounded treated & Mild & Low \\
Diabetic & No & Low \\
Diabetic treated & Low & Mild \\
Diabetic wounded & No & Low \\
Diabetic wounded treated & Low & Dense \\
\hline
\end{tabular}

Chung et al. [25] used a small molecule activator of Hsp72 to improve insulin sensitivity and inflammation in a genetic mouse model of insulin resistance, and Hsp72 also increases mitochondrial volume and improves metabolic homeostasis in a rat model of T2D [26]. It is likely, therefore, that when WP activates Hsp72, this improves insulin sensitivity and inflammation during diabetic wound healing. Our previous investigation [3] confirmed that WP was able to increase insulin concentration in blood of T1D. Improvements associated with Hsp72 are usually linked with a reduction in JNK1 phosphorylation, as has been previously confirmed by Darren et al. [26]. Hsp72 expression is decreased in T2D [25, 26] because meta-inflammation disrupts insulin signalling. Hsps, therefore, appear to have the potential to inhibit inflammatory kinases. This previous data suggests that improvement of insulin through WP is mediated by Hsp up-regulation. Interestingly, here, Hsp72 in the pancreas was found to show dense reactivity in the WP-treated diabetic wound group.

Furthermore, we found a clear correlation between WP associated up-regulation of Krt16 at day 4 (an indicator of epidermal hyperplasia and keratinocyte migration), and the complete and normal healing of diabetic wounds. In contrast, when the experimentally challenged diabetic wounds delayed the expression of Krt16 to day 8, it failed to regulate the healing phases properly. This is because a lack of Krt16 leads to a failure to regulate the production of innate signals and an over-activation of the expression of cytokines and other regulators of skin barrier function 
[17]. Early activation of Krt16 expression after various types of injuries to the skin is therefore functionally relevant to the progression of cutaneous inflammation.

Krt16 was significantly inhibited in keratinocytes of diabetic wounds. In [27], the inhibition of Krt16 coincided with the induction of Smad2 in the corresponding epithelia. Thus, Krt16 was inhibited in keratinocytes in diabetic wounds due to the up-regulation of Smad2 expression, which is indeed increased in diabetes [28]. Furthermore, it was concluded that puerarin exerted its anti-diabetic effect on STZ-treated rats through the inhibition of the TGF- $\beta 1 / \mathrm{Smad} 2$ pathway [28]. It is likely that WP exerted its anti-diabetic effect on the STZ-treated rats in this study through the same pathway, and that this served to increase Krt16, which is important for keratinocyte migration and epidermal hyperplasia. This suggested mechanism could explain the normalizing effect of WP in the diabetic wounds mediated by Krt16. Thus, loss of Krt16 at the optimal time checkpoint in diabetic wounds may impair the inflammatory stage resulting in inappropriate immune responses [17].

\section{Conclusions}

Our data confirms a role for Krt16 in the improvement of the inflammatory phase in the diabetic wounds, thereby leading to successful healing. In addition, WP was able to stimulate Hsp72 expression in early phases of wound healing in diabetic rats, resulting in a more normal healing process. From a therapeutic point of view, it is important to determine a protein by which Hsp72 and Krt16 can be induced. However, further studies are also warranted to investigate the role of WP as a potential future pharmacological approach to combat metabolic diseases.

\section{Abbreviations}

CMC: carboxymethyl cellulose; Hsp72: heat shock protein-72; HE: hematoxylin and eosin; ROS: reactive oxygen species; Krt16: keratin 16; STZ: streptozotocin; TNF-a: tumor necrosis factor-alpha;T1D: type 1 diabetes; T2D: type 2 diabetes; WP: whey protein.

\begin{abstract}
Authors' contributions
RA carried out the immunohistochemical studies, drafted the results and methods. AM carried out the animal model. OM participated in the design of the study and performed the statistical analysis. AM extracted the whey protein. HE designed this study and drafted the introduction and the discussion sections and prepared the manuscript for submission. All authors read and approved the final manuscript.
\end{abstract}

\footnotetext{
Author details

${ }^{1}$ Cell Biology and Histology Division, Zoology Department, Faculty of Science, Beni-Suef University, Beni-Suef, Egypt. ${ }^{2}$ Physiology Division, Zoology Department, Faculty of Science, Beni-Suef University, Beni-Suef, Egypt. ${ }^{3}$ Department of Food Science, College of Agriculture and Food Science, King Saud University, Riyadh, Saudi Arabia. ${ }^{4}$ Department of Dairy, Faculty of Agriculture, El-Minia University, El-Minia, Egypt. ${ }^{5}$ Department of Zoology, College of Science, King Saud University, P.O. Box 2455, Riyadh 11451 , KSA. ${ }^{6}$ Department of Zoology, Faculty of Science, El-Minia University, El-Minia, Egypt.
}

\section{Acknowledgements}

This project was funded by the National Plan for Sciences, Technology and Innovation (MAARIFAH), King Abdualaziz City for Science and Technology, Kingdom of Saudi Arabia, Award Number (12-BIO-2483-02).

\section{Compliance with ethical guidelines}

\section{Competing interests}

The authors declare that they have no competing interests.

Received: 23 April 2015 Accepted: 17 September 2015

Published online: 01 October 2015

\section{References}

1. Ebaid H, Salem A, Sayed A, Metwalli A. Whey protein enhances normal inflammatory responses during cutaneous wound healing in diabetic rats. Lipids Health Dis. 2011;10:235.

2. Ebaid H, Ahmed O, Mahmoud A, Ahmed R. Limiting prolonged inflammation during proliferation and remodeling phases of wound healing in streptozotocin-induced diabetic rats supplemented with camel undenatured whey protein. BMC Immunol. 2013;14:31.

3. Ebaid H. Promotion of immune and glycaemic functions in streptozotocin-induced diabetic rats treated with un-denatured camel milk whey proteins. Nutr Metab Lond. 2014;11:31.

4. Jiang B, Liang P, Deng G, Tu Z, Liu M, Xiao X. Increased stability of BCl-2 in HSP70-mediated protection against apoptosis induced by oxidative stress. Cell Stress Chaperones. 2011;16(2):143-52.

5. Dal-Pizzol F. Alternative activated macrophage: a new key for systemic inflammatory response syndrome and sepsis treatment? Crit Care Med. 2004;32(9):1971-2.

6. Sadique MZ, Grieve R, Harrison DA, Cuthbertson BH, Rowan KM. Is Drotrecogin alfa (activated) for adults with severe sepsis, cost-effective in routine clinical practice? Crit Care. 2011;15(5):R228.

7. Park H, Ssang-Goo C., Chang KK., Hyun SH., Kyung TN, Mi-Sung K, SungHo H, Myung JK, Kanghyun R, Eun KK, et al. Heat shock protein Hsp72 is a negative regulator of apoptosis signal-regulating kinase 1 . Mol Cell Biol. 2002:22:7721-30

8. Carcillo J, Holubkov R, Dean JM, et al. Rationale and design of the pediatric critical illness stress-induced immune suppression (CRISIS) prevention trial. J Parenter Enter Nutr. 2009;33(4):368-74.

9. Gabai VL, Meriin AB, Mosser D, Caron AW, Rits S, Shifrin VI, Sherman MY. Hsp70 prevents activation of stress kinases: a novel pathway of cellular thermotolerance. J Biol Chem. 1997;272:18033-7.

10. Meriin AB, Gabai VL, Yaglom J, Shifrin VI, Sherman MY. Proteasome inhibitors activate stress kinases and induce Hsp72. Diverse effects on apoptosis. J Biol Chem. 1998;273:6373-9.

11. Saleh A, Srinivasula SM, Balkir L, Robbins PD, Alnemri ES. Negative regulation of the Apaf-1 apoptosome by Hsp70. Nat Cell Biol. 2000;2:476-83.

12. Park HS, Lee JS, Huh SH, Seo JS, Choi EJ. Hsp72 functions as a natural inhibitory protein of c-Jun N-terminal kinase. EMBOJ. 2001;20:446-56.

13. Fuchs E, Weber K. Intermediate filaments: structure, dynamics, function, and disease. Annu Rev Biochem. 1994;63:345-82.

14. Komine M, Rao LS, Kaneko T, Tomic CM, Tamaki K, Freedberg IM, Kuroda K, Tajima S. HSP47 is a useful marker for skin fibroblasts in formalin-fixed, paraffin-embedded tissue specimens. J Cutan Pathol. 2004;31(3):241-6

15. Rotty JD, Coulombe PA. A wound-induced keratin inhibits Src activity during keratinocyte migration and tissue repair. J Cell Biol. 2012;197(3):381-9.

16. Haider AS, Peters SB, Kaporis H, Cardinale I, Fei J, Ott J, Blumenberg M, Bowcock AM, Krueger JG, Carucci JA. Genomic analysis defines a cancerspecific gene expression signature for human squamous cell carcinoma and distinguishes malignant hyperproliferation from benign hyperplasia. J Invest Dermatol. 2006;126(4):869-81.

17. Juliane $C L$, Sylvia P, Jeremy DR, Robyn PH, Roger LC, Allan B, Pierre AC. Keratin 16 regulates innate immunity in response to epidermal barrier breach. PNAS. 2013;110(48):19537-42. 
18. Ebaid $\mathrm{H}$. Neutrophil depletion in the early inflammatory phase delayed cutaneous wound healing in older rats: improvements due to the use of un-denatured camel whey protein. Diagn Pathol. 2014;9(1):46.

19. Souil E, Capon A, Mordon S, Nh-Xuan AT, Polla BS, Bachelet M. Treatment with 815-nm diode laser induces long-lasting expression of 72-kDa heat shock protein in normal rat skin. Br J Dermatol. 2001;144(2):260-6.

20. Khanra R, Dewanjee S, Dua TK, Sahu R, Gangopadhyay M, DeFeo V, Zia-UIHaq M. Abroma augusta L. (Malvaceae) leaf extract attenuates diabetes induced nephropathy and cardiomyopathy via inhibition of oxidative stress and inflammatory response. J Translational Med. 2015;13:1-14.

21. Krifa M, Bouhlel I, Ghedira-chekir L, Ghedira K. Immunomodulatory and cellular anti-oxidant activities of an aqueous extract of Limoniastrum guyonianum gall. J Ethnopharmacol. 2013; 2: S0378-8741.

22. Keagle JN, Welch WJ, Young DM. Expression of heat shock proteins in a linear rodent wound. Wound Repair Regen. 2001;9(5):378-85.

23. Helbig D, Bodendorf MO, Grunewald S, Kendler M, Simon JC, Paasch U. Immunohistochemical investigation of wound healing in response to fractional photothermolysis. J Biomed Opt. 2009;14(6):064044.
24. Calapre L, Gray ES, Ziman M. Heat stress: a risk factor for skin carcinogenesis. Cancer Lett. 2013;337(1):35-40.

25. Chung J, Nguyen AK, Henstridge DC, Holmes AG, Chan MH, Mesa JL, et al. HSP72 protects against obesity-induced insulin resistance. Proc Nat Acad Sci USA. 2008;105:1739-44.

26. Darren $\mathrm{CH}$, Martin W, Mark AF. Chaperoning to the metabolic party: the emerging therapeutic role of heat-shock proteins in obesity and type 2 diabetes. Mol Metab. 2014;3:781-93.

27. Tomikawa K, Yamamoto T, Shiomi N, Shimoe M, Hongo S, Yamashiro K, Yamaguchi T, Maeda H, Takashiba S. Smad2 decelerates re-epithelialization during gingival wound healing. J Dent Res. 2012;91(8):764-70.

28. She S, Liu W, Li T, Hong Y. Effects of puerarin in STZ-induced diabetic rats by oxidative stress and the TGF- $\beta 1 /$ Smad 2 pathway. Food Funct. 2014;5(5):944-50.

\section{Submit your next manuscript to BioMed Central and take full advantage of:}

- Convenient online submission

- Thorough peer review

- No space constraints or color figure charges

- Immediate publication on acceptance

- Inclusion in PubMed, CAS, Scopus and Google Scholar

- Research which is freely available for redistribution

Submit your manuscript at

www.biomedcentral.com/submit

C Biomed Central 